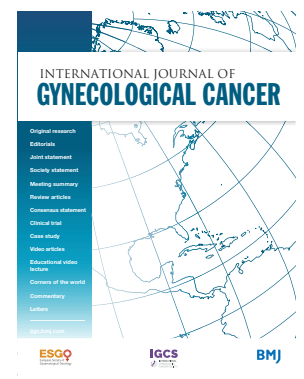

\title{
Ultrasound, macroscopic and histological features of malignant ovarian tumors
}

Francesca Moro (D) , ${ }^{1}$ Valeria Verdecchia, ${ }^{1,2}$ Paola Romeo, ${ }^{1}$ Francesca Ciccarone,${ }^{1}$ Gian Franco Zannoni, ${ }^{1}$ Lil Valentin, ${ }^{3,4}$ Dirk Timmerman, ${ }^{5}$ Tom Bourne,${ }^{6,7}$ Wouter Froyman, ${ }^{5}$ Giovanni Scambia, ${ }^{1,8}$ Antonia Carla Testa ${ }^{1,8}$

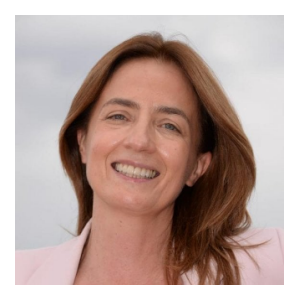

\section{Antonia Carla Testa}

${ }^{1}$ Dipartimento Scienze della Salute della Donna, del Bambino e di Sanità Pubblica, Fondazione Policlinico Universitario Agostino Gemelli, IRCCS, Rome, Italy

${ }^{8}$ Dipartimento Scienze della Vita e Sanità Pubblica, Università Cattolica del Sacro Cuore, Rome, Italy

Biography: Professor Antonia Carla Testa is a member of the IOTA steering committee. She is director of a School of Ultrasound in Gynecology at Policlinico Universitario Agostino Gemelli, IRCCS in Rome.
For numbered affiliations see end of article.

\section{Correspondence to} Dr Francesca Moro, Dipartimento Scienze della Salute della Donna, del Bambino e di Sanità Pubblica, Fondazione Policlinico Universitario Agostino Gemelli, IRCCS, Rome, Italy; morofrancy@gmail.com

Accepted 6 April 2020 Published Online First 20 August 2020

\section{SUMMARY}

Ultrasound examination is considered to be the first line imaging method to diagnose an ovarian mass with a high degree of accuracy, discriminating between benign and malignant ovarian masses in the hands of experienced examiners.

The International Ovarian Tumor Analysis (IOTA) group provided a standardized terminology of ovarian masses ${ }^{1}$ and suggested simple ultrasound rules that can be used to classify adnexal masses as benign or malignant. ${ }^{2}$ The IOTA group has also created logistic regression models (ie, ADNEX
(Assessment of Different NEoplasias in the adneXa) model), including clinical and ultrasound information to calculate the likelihood of malignancy in adnexal masses. The IOTA ADNEX model estimates the likelihood not only of an adnexal mass being benign or malignant but also the likelihood that the mass is benign, borderline malignant, stage I primary invasive malignant, stage II-IV primary invasive malignant or a metastasis in the ovary from another primary tumor. ${ }^{3}$

Recently, a consensus meeting including European and North American professionals developed

\section{ULTRASOUND, MACROSCOPIC AND HISTOLOGICAL FEATURES OF MALIGNANT OVARIAN TUMORS:}

\section{REPRESENTATIVL EO CASE SERIES}

\section{Check for updates}

(C) IGCS and ESG0 2021. No commercial re-use. See rights and permissions. Published by BMJ.

To cite: Moro F, Verdecchia V, Romeo P, et al. Int J Gynecol Cancer 2021;31:150-151. 


\section{Educational video lecture}

a new risk model for the pre-operative assessment of adnexal masses, called 0-RADS (Ovarian-Adnexal Reporting and Data System). The 0-RADS ultrasound risk stratification and management system was designed to provide consistent interpretations, to decrease or eliminate ambiguity in ultrasound reports resulting in a higher probability of accuracy in assigning risk of malignancy to ovarian and other adnexal masses, and to provide a management recommendation for each risk category. ${ }^{4}$

Moreover, during the past 15 years authors of the IOTA group have described the typical ultrasound appearance of several different adnexal pathologies, including various histotypes of malignancy, collected in the series of "Imaging in gynecology" papers. ${ }^{5-10}$ Indeed, ovarian cancer includes several histological entities which can be grouped into four histological groups: epithelial tumors, germ cell tumors, stromal tumors and metastatic tumors. Each histopathological category is often characterized by some morphological typical features, as described in the textbooks of pathologists.

In the pre-operative phase, ultrasound examination can enable assessment of these macroscopic aspects, thus providing a presumptive histological diagnosis.

We present a video describing how to apply the simple ultrasound rules, the IOTA ADNEX model and the 0-RADS model, and provides explanatory examples for each model.

\section{Author affiliations} \\ ${ }^{1}$ Dipartimento Scienze della Salute della Donna, del Bambino e di Sanità Pubblica, \\ Fondazione Policlinico Universitario Agostino Gemelli, IRCCS, Rome, Italy \\ ${ }^{2}$ Dipartimento di Scienze Cliniche Specialistiche ed Odontostomatologiche, \\ Università Politecnica delle Marche, Ancona, Italy \\ ${ }^{3}$ Department of Obstetrics and Gynecology, Skåne University Hospital, Malmö, \\ Sweden \\ ${ }^{4}$ Department of Clinical Sciences Malmö, Lund University, Malmö, Sweden \\ ${ }^{5}$ Department of Obstetrics and Gynecology, Multidisciplinary Breast Centre and \\ Leuven University Centre for Cancer Prevention (LUCK), University Hospitals \\ Leuven, Leuven, Belgium \\ ${ }^{6}$ Department of Obstetrics and Gynaecology, Queen Charlotte's and Chelsea \\ Hospital, Imperial College London, London, UK \\ ${ }^{7}$ Department of Development \& Regeneration, KU Leuven, Leuven, Belgium \\ ${ }^{8}$ Dipartimento Scienze della Vita e Sanità Pubblica, Università Cattolica del Sacro \\ Cuore, Rome, Italy
}

Contributors We present a video describing how to apply the simple ultrasound rules, the IOTA ADNEX model and the 0-RADS model, and explanatory examples for each model.

Funding The authors have not declared a specific grant for this research from any funding agency in the public, commercial or not-for-profit sectors.

Competing interests None declared.

Patient consent for publication Not required.

Provenance and peer review Commissioned; internally peer reviewed.

ORCID iD

Francesca Moro http://orcid.org/0000-0002-5070-7245

\section{REFERENCES}

1 Timmerman D, Valentin L, Bourne TH, et al. Terms, definitions and measurements to describe the sonographic features of adnexal tumors: a consensus opinion from the International Ovarian Tumor Analysis (IOTA) Group. Ultrasound Obstet Gynecol 2000;16:500-5.

2 Timmerman D, Testa AC, Bourne T, et al. Simple ultrasound-based rules for the diagnosis of ovarian cancer. Ultrasound Obstet Gynecol 2008;31:681-90.

3 Van Calster B, Van Hoorde K, Valentin L, et al. Evaluating the risk of ovarian cancer before surgery using the ADNEX model to differentiate between benign, borderline, early and advanced stage invasive, and secondary metastatic tumours: prospective multicentre diagnostic study. BMJ 2014;349:g5920.

4 Andreotti RF, Timmerman D, Strachowski LM, et al. O-RADS US risk stratification and management system: a consensus guideline from the ACR Ovarian-Adnexal Reporting and Data System Committee. Radiology 2020;294:168-85.

5 Fruscella E, Testa AC, Ferrandina G, et al. Ultrasound features of different histopathological subtypes of borderline ovarian tumors. Ultrasound Obstet Gynecol 2005;26:644-50.

6 Testa AC, Ferrandina G, Timmerman D, et al. Imaging in gynecological disease (1): ultrasound features of metastases in the ovaries differ depending on the origin of the primary tumor. Ultrasound Obstet Gynecol 2007;29:505-11.

7 Moro F, Zannoni GF, Arciuolo D, et al. Imaging in gynecological disease (11): clinical and ultrasound features of mucinous ovarian tumors. Ultrasound Obstet Gynecol 2017;50:261-70.

8 Moro F, Baima Poma C, Zannoni GF, et al. Imaging in gynecological disease (12): clinical and ultrasound features of invasive and non-invasive malignant serous ovarian tumors. Ultrasound Obstet Gynecol 2017;50:788-99.

9 Moro F, Magoga G, Pasciuto T, et al. Imaging in gynecological disease (13): clinical and ultrasound characteristics of endometrioid ovarian cancer. Ultrasound Obstet Gynecol 2018;52:535-43.

10 Pozzati F, Moro F, Pasciuto T, et al. Imaging in gynecological disease (14): clinical and ultrasound characteristics of ovarian clear cell carcinoma. Ultrasound Obstet Gynecol 2018;52:792-800. 